\title{
PTEN loss is associated with a poor response to trastuzumab in HER2-overexpressing gastroesophageal adenocarcinoma
}

\author{
Yasunori Deguchi $^{1} \cdot$ Hiroshi Okabe ${ }^{1,4} \cdot$ Nobu Oshima $^{1} \cdot$ Shigeo Hisamori $^{1} \cdot$ \\ Sachiko Minamiguchi ${ }^{2} \cdot$ Manabu Muto $^{3} \cdot$ Yoshiharu Sakai $^{1}$
}

Received: 18 February 2016/Accepted: 2 August 2016/Published online: 12 August 2016

(c) The International Gastric Cancer Association and The Japanese Gastric Cancer Association 2016

\begin{abstract}
Background Although trastuzumab improves the outcome of patients with human epidermal growth factor receptor 2 (HER2)-overexpressing gastric or gastroesophageal junction adenocarcinoma (collectively referred to as "gastroesophageal adenocarcinoma"; GEA), no clinical response is observed in a substantial population of patients. A predictive biomarker of trastuzumab response is required. The aim of this study was to evaluate whether the hyperactivation of the downstream phosphatidylinositol 3-kinase pathway, due to phosphatase and tensin homolog (PTEN) loss or PIK3CA mutations, could provide trastuzumab resistance in GEA.

Methods Expression of HER2 and PTEN, and PIK3CA gene mutations were screened in 264 surgically resected GEA specimens. The effects of PTEN knockdown on the response to trastuzumab on cell viability, HER2 downstream signaling, apoptosis, and cell cycle were evaluated in HER2-overexpressing NCI-N87 gastric adenocarcinoma and OE19 esophageal adenocarcinoma cell lines. Inhibition of xenograft tumor growth by trastuzumab was investigated in OE19 cells with or without PTEN knockdown. The
\end{abstract}

Hiroshi Okabe

hokabe@kuhp.kyoto-u.ac.jp

1 Department of Surgery, Graduate School of Medicine, Kyoto University, 54 Shogoin Kawahara-cho, Sakyo-ku, Kyoto 606-8507, Japan

2 Department of Diagnostic Pathology, Graduate School of Medicine, Kyoto University, Kyoto, Japan

3 Department of Therapeutic Oncology, Graduate School of Medicine, Kyoto University, Kyoto, Japan

4 Department of Surgery, Otsu Municipal Hospital, 2-9-9 Motomiya, Otsu, Shiga 520-0804, Japan
PTEN expression and objective response were analyzed in 23 GEA patients who received trastuzumab-based therapy. Results PTEN loss was identified in $34.5 \%$ of HER2overexpressing GEA patients, whereas PIK3CA mutations were rare $(5.6 \%)$. Trastuzumab-mediated growth suppression, apoptosis, and $\mathrm{G}_{1}$ cell cycle arrest were inhibited by PTEN knockdown through Akt activation in NCI-N87 and OE19 cells. PTEN knockdown impaired the antiproliferative effect of trastuzumab in OE19 xenograft models. A clinical response was observed in $50 \%$ of PTEN-positive tumors (9 of 18) but in no tumors with PTEN loss (none of 5).

Conclusions PTEN loss was frequently found in HER2overexpressing tumors, and was associated with a poor response to trastuzumab-based therapy in patients with GEA.

Keywords Gastric adenocarcinoma - Esophageal adenocarcinoma - Phosphatase and tensin homolog . Human epidermal growth factor receptor 2 . Trastuzumab

\section{Introduction}

Gastric cancer is the fifth commonest malignancy and the third leading cause of cancer death in the world [1]. Most gastric cancer patients have highly advanced disease at presentation, despite improvements in the diagnosis, and thus the treatment option is mainly limited to systemic chemotherapy. Patients with advanced gastric cancer rarely achieve long-term survival, and the median survival is 8-14 months [2, 3]. In addition, the incidence of gastroesophageal junction adenocarcinoma has increased considerably in the past 40 years, especially in industrialized countries [4]. 
The Trastuzumab for Gastric Cancer (ToGA) trial, a recent international randomized controlled trial, demonstrated a significant survival benefit of trastuzumab combined with chemotherapy for human epidermal growth factor receptor 2 (HER2)-overexpressing gastric or gastroesophageal junction adenocarcinoma (collectively referred to as "gastroesophageal adenocarcinoma"; GEA) [5]. However, more than half of the patients in the ToGA trial did not exhibit an objective response to trastuzumab, despite HER2 overexpression. Moreover, trastuzumab has several unique adverse effects, such as cardiotoxicity, which causes severe cardiac dysfunction in 0-3.9\% of patients [6]. Therefore, a predictive biomarker is required to select patients who will likely benefit from trastuzumabbased therapy.

The phosphatidylinositol 3-kinase (PI3K) pathway is a major downstream signal transduction pathway of HER2. In breast cancer, constitutive activation of the PI3K pathway due to phosphatase and tensin homolog (PTEN) deficiency or phosphatidylinositol 4,5-bisphosphate 3-kinase catalytic subunit alpha gene (PIK3CA) mutations was identified to be one of the important mechanisms of trastuzumab resistance [7-9].

However, the mechanisms of trastuzumab resistance in GEA have yet to be clarified. We hypothesized that the hyperactivation of the PI3K pathway might contribute to trastuzumab resistance also in GEA. The first aim of this study was to evaluate the frequency of PTEN loss and PIK3CA mutations, and to evaluate the relationship between alterations in the PI3K pathway and HER2 overexpression in GEA. The second aim was to explore whether PTEN loss or PIK3CA mutation is associated with trastuzumab resistance and influences the response to trastuzumab therapy in patients with GEA.

\section{Materials and methods}

\section{Patients}

Two separate cohorts of patients were investigated in the study. First, 326 patients who underwent R0 curative resection for primary GEA at Kyoto University Hospital between January 2001 and December 2010 received a diagnosis of pathologic T2-T4 and pathologic TNM stage IB-IIIC cancer (seventh edition of the Union for International Cancer Control TNM classification). Sixty-two patients received preoperative chemotherapy and were excluded from the study. Therefore, surgical specimens from 264 patients were screened for HER2 expression, PTEN expression, and PIK3CA mutation status (screening cohort).
The second cohort included 24 patients with HER2overexpressing advanced GEA who received trastuzumabbased therapy from November 2010 to December 2014 at Kyoto University Hospital. Specimens for HER2 and PTEN evaluation were obtained by biopsy or surgical resection, but a specimen was not available in one patient. Therefore, 23 patients were included in the study to investigate the influence of PTEN loss on trastuzumab sensitivity (investigation cohort). Trastuzumab was administered to these patients with oral fluoropyrimidine drugs (capecitabine or S-1) with or without cisplatin. The objective response was assessed by the Response Evaluation Criteria in Solid Tumors, version 1.1 [10].

All patients gave informed consent for additional molecular analyses at the time of biopsy or surgery, and formalin-fixed paraffin-embedded tissue specimens were obtained under the protocol approved by the Institutional Review Board of Kyoto University (E1752). Clinicopathology information was retrieved from medical records and pathology reports.

\section{Evaluation of HER2 expression and amplification}

HER2 expression was analyzed by immunohistochemistry (IHC), and ERBB2 amplification was evaluated by dualcolor in situ hybridization (DISH) as previously described [11]. Briefly, IHC staining of HER2 with the PATHWAY ${ }^{\circledR}$ HER2/neu (4B5) antibody (Ventana Medical Systems) was performed with an automated slide staining system (BenchMark XT; Ventana Medical Systems). The scoring system defined in the ToGA study was used for IHC scoring (evaluated by membranous reactivity in $10 \%$ or more of tumor cells.) [5]. DISH was performed with an INFORM DISH HER2 kit (Ventana Medical Systems). The post hoc analysis of the ToGA study showed that IHC score $0 / 1+$ patients with ERBB2 amplification did not benefit from trastuzumab-based chemotherapy [5]. Therefore, in this study, HER2 overexpression was defined as either IHC score $3+$ or IHC score $2+$ with DISH positivity.

\section{Evaluation of PTEN expression}

The evaluation of PTEN expression was performed by IHC with a PTEN monoclonal antibody (clone 138G6, \#9559, Cell Signaling Technology, Danvers, MA, USA; diluted $1: 200)$ according to the manufacturer's protocol. PTEN IHC was subjectively scored as absent (0) if no immunostaining was detectable in cancer cells, as weak $(1+)$ if cytoplasmic staining was low, as moderate $(2+)$ if cytoplasmic staining was intermediate between weak and strong, and as strong $(3+)$ if the cytoplasmic staining was intense (Fig. 1). PTEN loss was determined when there was negative staining (score 0 ) of cells in more than $75 \%$ 

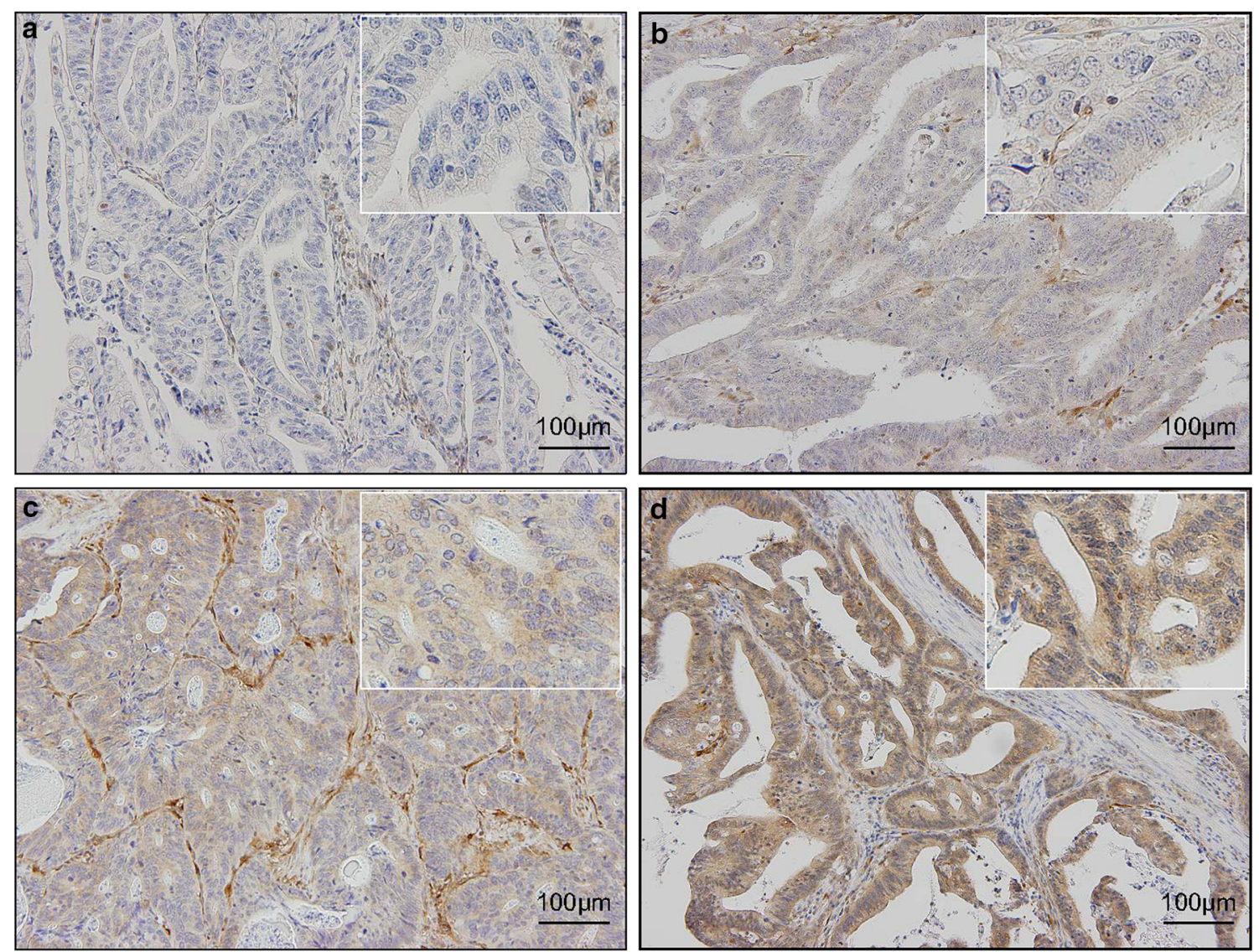

Fig. 1 Phosphatase and tensin homolog (PTEN) expression in gastroesophageal adenocarcinoma specimens. Representative PTEN scoring by immunohistochemistry. Immunoreactivity of adjacent stromal cells was used as an internal positive control. a Score 0: no immunostaining is detectable within the tumor cells. b Score 1: the

of the tumor as previously reported [12]. A PTEN-positive status was defined as weak, moderate, or strong staining (score $1+$ to $3+$ ) of cells in more than $25 \%$ of the tumor. Interpretation was performed by two independent observers (Y.D. and S.M.), and discrepancies were discussed to obtain a final result.

\section{PIK3CA mutation analysis}

Samples from 97 of the 264 patients who underwent surgery before 2004 were not in the appropriate state for mutational analysis. DNA was successfully extracted from macrodissected tumor tissue (more than $75 \%$ tumor content) from the remaining 167 patients, with a NucleoSpin DNA FFPE XS kit (Macherey-Nagel, Germany) according to the manufacturer's instructions. Mutation analysis of the hot spot regions of PIK3CA (exons 9 and 20) was performed by PCR amplification followed by direct sequencing with a 3130xl Genetic Analyzer (Applied Biosystems, USA).

immunostaining is weak. c Score 2: the immunostaining is intermediate between weak and strong. d Score 3: strong immunostaining. PTEN loss was defined as negative staining (score 0 ) of cells in more than $75 \%$ of the tumor (a), and the specimens in $\mathbf{b}-\mathbf{d}$ were evaluated as PTEN positive. Scale bars represent $100 \mu \mathrm{m}$

\section{Cell lines, cell culture, and reagents}

The human gastric adenocarcinoma cell lines NCI-N87, AGS, KATO-III, and SNU-1 were purchased from American Type Culture Collection (Manassas, VA, USA), and the MKN1, MKN45, MKN7, and MKN74 cell lines were purchased from RIKEN BRC Cell Bank (Ibaraki, Japan). The human esophageal adenocarcinoma cell lines OE19 and OE33 and the gastric adenocarcinoma cell line HGC27 were purchased from the European Collection of Cell Cultures. All cell lines were cultured in RPMI 1640 medium supplemented with $10 \%$ fetal bovine serum (Life Technologies, Carlsbad CA, USA), penicillin (100 U/ml) and streptomycin $(100 \mu \mathrm{g} / \mathrm{ml}$; Life Technologies), and were incubated at $37{ }^{\circ} \mathrm{C}$ in a humidified chamber containing $5 \% \mathrm{CO}_{2}$.

Trastuzumab was kindly provided by Chugai Pharmaceutical (Tokyo, Japan) for nonclinical investigations. Anti-HER2 Affibody ${ }^{\circledR}$ molecule (fluorescein isothiocyanate) (ab31891) was purchased from AbCam 
(Cambridge, MA, USA) for flow cytometry analysis of membranous HER2 expression.

\section{Small interfering RNA and short hairpin RNA}

Two distinct small interfering RNA (siRNA) species targeting PTEN (siPTEN \#1, Hs_PTEN_6 FlexiTube siRNA, SI00301504; siPTEN \#2, Hs_PTEN_8 FlexiTube siRNA, SI03048178) and nonsilencing control siRNA (AllStars negative control siRNA, SI03650318) were purchased from Qiagen, and were transfected with use of Lipofectamine RNAiMAX (Invitrogen Life Technologies, Carlsbad, CA, USA) according to the manufacturer's reverse-transfection protocol. Briefly, siRNA at a final concentration of $3 \mathrm{mM}$ with Lipofectamine RNAiMAX in Opti-MEM was first added to wells or dishes and incubated at room temperature for $30 \mathrm{~min}$, and then cells were seeded. Cells were incubated overnight and the culture medium was changed to fresh medium or medium containing trastuzumab at $10 \mu \mathrm{g} /$ $\mathrm{ml}$.

The control short hairpin RNA (shRNA) vector (pLKO.1 scrambled shRNA), packaging vector (psPAX2, plasmid 12260), and envelope vector (pMD2.G, plasmid 12259) were purchased from Addgene (Cambridge, MA, USA). The shRNA sequences targeting PTEN were made as the same sequences as the purchased siRNA (shPTEN \#1 as siPTEN \#1 and shPTEN \#2 as siPTEN \#2), and cloned into pLKO.1 vectors. Lentivirus containing shRNA constructs was made according to the manufacturer's protocol, and cell lines were infected with it. We selected stable knockdown clones by culturing cells with puromycin ( $2 \mu \mathrm{g} / \mathrm{ml})$ for at least 4 weeks.

\section{Western blotting}

Cells were washed with ice-cold phosphate-buffered saline and lysed in sodium dodecyl sulfate lysis buffer supplemented with protease inhibitor cocktail (Nacalai Tesque, Kyoto, Japan) and phosphatase inhibitor cocktail (Nacalai Tesque, Kyoto, Japan). The protein concentration was determined with a bicinchoninic acid protein assay kit (Thermo Scientific, Rockford IL, USA). A total of $20 \mu \mathrm{g}$ of whole cell lysate were subjected to sodium dodecyl sulfate-polyacrylamide gel electrophoresis and transferred to a poly(vinylidene difluoride) membrane (Merck Millipore). Membranes were probed with specific primary antibodies against HER2 (polyclonal, \#2242), PTEN (clone 138G6, \#9559), pan-Akt (clone C67E7, \#4691), phosphorylated Akt (Ser473, clone D9E, \#4060), p44/p42 mitogen-activated protein kinase [MAPK; extracellular-signal-regulated kinase (ERK) 1/2] (clone 137F5, \#4695), and phosphorylated p44/p42 MAPK (ERK1/2) (Thr202/Tyr204, clone D13.14.4E, \#4370) (Cell Signaling Technology, Danvers,
MA, USA) and with horseradish peroxidase (HRP)-conjugated secondary antibody (Dako). HRP-conjugated $\beta$ actin antibody (Sigma) was used as a loading control. Bands were visualized with use of a Pierce Western blotting substrate kit (Thermo Scientific).

\section{Cell viability and cell growth inhibition assay}

The cell viability was measured by the WST- 8 colorimetric assay [Cell Counting Kit-8 (CCK-8); Dojindo, Kumamoto, Japan]. Exponentially growing cells $\left(2 \times 10^{3}-10 \times 10^{3} /\right.$ $100 \mu \mathrm{l} /$ well) were seeded in triplicate into 96-well plates. The cells were not treated with trastuzumab or were treated with trastuzumab for $72 \mathrm{~h}$, and $10 \mu \mathrm{l}$ of CCK- 8 was added to each well and the plates were further incubated for $3 \mathrm{~h}$. The absorbance at 450 and $600 \mathrm{~nm}$ was measured with a GloMax-Multi detection system (Promega) to calculate the number of viable cells in each well. Cell growth inhibition (\%) was calculated with the following formula: [1 - experimental absorbance (treated well)/control absorbance (untreated well)] $\times 100$.

\section{Apoptosis assay}

Exponentially growing cells $\left(5 \times 10^{3}-10 \times 10^{3} / 100 \mu \mathrm{l} /\right.$ well) were plated in triplicate in 96-well plates. The cells were not treated with trastuzumab or were treated with trastuzumab for $72 \mathrm{~h}$, and caspase 3/7 activity was measured with a Caspase-Glo 3/7 assay kit (Promega) according to the manufacturer's protocol. Luminescence was measured with the GloMax-Multi detection system (Promega). Caspase activity was normalized to the cell number obtained from the CCK- 8 assay performed simultaneously under the same conditions [13].

\section{Cell cycle analysis}

The cells were dissociated with trypsin/EDTA, washed with phosphate-buffered saline, and fixed with ice-cold $70 \%$ ethanol at $-20{ }^{\circ} \mathrm{C}$ overnight. The cells were treated with ribonuclease I (Nacalai Tesque) for $10 \mathrm{~min}$ at $37{ }^{\circ} \mathrm{C}$ to digest RNA and were stained with propidium iodide (100 $\mu \mathrm{g} / \mathrm{ml}$; Sigma). The cells were analyzed by flow cytometry (BD Accuri $^{\mathrm{TM}}$ C6 flow cytometer).

\section{Xenograft experiments}

Six-week-old female KSN/slc athymic nude mice (Japan SLC, Shizuoka, Japan) were maintained under specific pathogen-free conditions. A total of $3 \times 10^{6}$ scrambled or stable PTEN-knockdown OE19 cells in $100 \mu \mathrm{l}$ of phosphate-buffered saline were injected subcutaneously into three or four points on the back. Treatment with 
intraperitoneal injection of trastuzumab $(30 \mathrm{mg} / \mathrm{kg}$ as a loading dose, then $15 \mathrm{mg} / \mathrm{kg}$ weekly) or normal saline was started 2 weeks after inoculation, when tumors became palpable and measurable. The tumor volume was estimated by the following formula: $0.5 \times L \times W \times W$, where $L$ is length and $W$ is width. The experiments were approved by the institutional Animal Ethics and Research Committee (MedKyo15513).

\section{Statistical analysis}

All values were expressed as the mean \pm standard deviation. All in vitro experiments were repeated at least three times. Statistical analyses were performed with JMP Pro 11 (SAS). Student's $t$ test was used to compare continuous variables. Dunnett's test was used for multiple comparisons. Categorical variables were analyzed by Fisher's exact test. Differences were considered statistically significant at a $p$ value less than 0.05 .

\section{Results}

\section{PTEN loss is frequent whereas PIK3CA mutation is rare in HER2-overexpressing GEA}

The expression/amplification of HER2, expression of PTEN, and mutations of the PIK3CA gene were screened in 264 patients with GEA. The characteristics of the screening cohort are shown in Table 1. The IHC scores for HER2 were $3+$ in 22 patients and $2+$ in 13 patients. A heterogeneous expression pattern of HER2 was often observed (Fig. 2a). DISH was positive in 7 of the 13 IHC score $2+$ patients. Therefore, 29 tumors $(11.0 \%)$ were defined as positive for HER2 overexpression (IHC score $3+$, or IHC score 2+ and DISH positive; Table 1).

Expression of PTEN was evaluated by IHC with use of the scoring system described in "Materials and methods" (Fig. 1). PTEN loss was found in 62 patients $(23.5 \%$; Table 1). Homogeneous expression of PTEN (90\% or more of tumor cells) was observed in most PTEN-positive cases (Fig. 2b).

The mutation status of the PIK3CA gene was successfully evaluated in 167 patients, and 17 PIK3CA mutations were identified in 16 patients (9.6\%; Table 1): 6 mutations in exon 9 (E545K in five cases and E545A in one case), and 11 mutations in exon 20 (H1047R). One patient had mutations in both exon 9 and exon 20. A comparison of the frequency of these alterations in relation to HER2 status revealed that the incidence of PTEN loss was higher in HER2-overexpressing tumors than in HER2-negative tumors, although the difference was not significant $(34.5 \%$ vs $22.1 \%, p=0.163$, Fisher's exact test; Table 1$)$. On the
Table 1 Characteristics of patients and tumors based on human epidermal growth factor receptor 2 (HER2) status

\begin{tabular}{|c|c|c|}
\hline \multirow[t]{2}{*}{ Variables } & \multicolumn{2}{|c|}{ HER2 overexpression } \\
\hline & Positive $(n=29)$ & Negative $(n=235)$ \\
\hline \multicolumn{3}{|l|}{ Age } \\
\hline Median (range) & $75(54-87)$ & $69(29-89)$ \\
\hline \multicolumn{3}{|l|}{ Sex } \\
\hline Male & $26(89.7 \%)$ & $155(66.0 \%)$ \\
\hline Female & $3(10.3 \%)$ & $80(34.0 \%)$ \\
\hline \multicolumn{3}{|l|}{ Tumor location } \\
\hline GE junction & $3(10.3 \%)$ & $18(7.7 \%)$ \\
\hline Stomach & $26(89.7 \%)$ & $217(92.3 \%)$ \\
\hline \multicolumn{3}{|c|}{ Lauren classification } \\
\hline Intestinal & $22(75.9 \%)$ & $79(33.6 \%)$ \\
\hline Diffuse & $1(3.4 \%)$ & $128(54.5 \%)$ \\
\hline Mixed & $6(20.7 \%)$ & $28(11.9 \%)$ \\
\hline \multicolumn{3}{|l|}{ pT category } \\
\hline 2 & $11(37.9 \%)$ & $89(37.9 \%)$ \\
\hline 3 & $12(41.4 \%)$ & $85(36.2 \%)$ \\
\hline 4 & $6(20.7 \%)$ & $61(26.0 \%)$ \\
\hline \multicolumn{3}{|l|}{ pN category } \\
\hline 0 & $7(24.1 \%)$ & $93(39.6 \%)$ \\
\hline $1-3$ & $22(75.9 \%)$ & $142(60.4 \%)$ \\
\hline \multicolumn{3}{|l|}{ pTNM stage } \\
\hline I & $3(10.3 \%)$ & $53(22.6 \%)$ \\
\hline II & $15(51.7 \%)$ & $89(37.9 \%)$ \\
\hline III & $11(37.9 \%)$ & $93(39.6 \%)$ \\
\hline \multicolumn{3}{|l|}{ PTEN expression } \\
\hline Loss & $10(34.5 \%)$ & $52(22.1 \%)$ \\
\hline Positive & $19(65.5 \%)$ & $183(77.9 \%)$ \\
\hline \multicolumn{3}{|l|}{ PIK3CA mutation } \\
\hline Present & $1(5.6 \%)^{\mathrm{a}}$ & $15(10.1 \%)^{\mathrm{a}}$ \\
\hline Absent & $17(94.4 \%)^{\mathrm{a}}$ & $134(89.9 \%)^{\mathrm{a}}$ \\
\hline Not evaluable & 11 & 86 \\
\hline
\end{tabular}

GE gastroesophageal, PTEN phosphatase and tensin homolog

${ }^{\text {a }}$ Percentage in evaluable samples

other hand, PIK3CA mutation was less frequent in both HER2-overexpressing tumors and HER2-negative tumors (5.6 \% vs $10.1 \%, p=1.000$, Fisher's exact test; Table 1 ).

\section{PTEN knockdown induces trastuzumab resistance in trastuzumab-sensitive cell lines}

The screening data suggested that PTEN loss is clinically more relevant in comparison with PIK3CA mutation. Therefore, we focused on PTEN deficiency, since this might have a major impact on trastuzumab resistance in GEA. Nine gastric and two esophageal adenocarcinoma cell lines were screened to evaluate HER2 and PTEN expression and trastuzumab sensitivity. Western blotting 
Fig. 2 Distribution of immunohistochemistry $(I H C)$ positive cells in tumors. a Distribution of human epidermal growth factor receptor 2 (HER2) IHC-positive $(2+$ and $3+)$ cells. The open bar indicates HER2 IHCnegative patients. b Distribution of phosphatase and tensin homolog (PTEN) IHC-positive (scores 1, 2, and 3) cells. Open bars indicate patients with PTEN loss

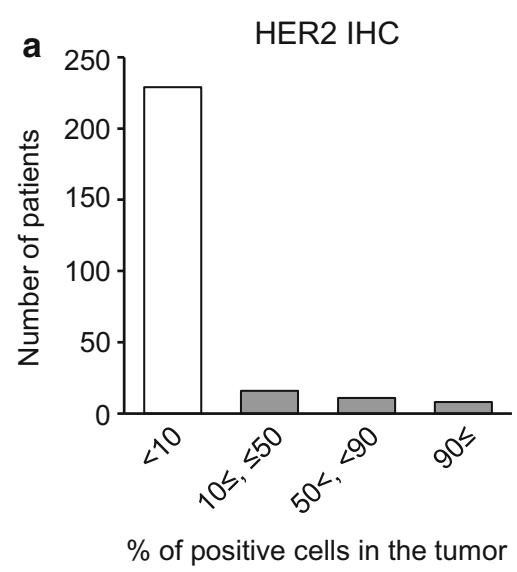

revealed that the NCI-N87 gastric adenocarcinoma cell line and the OE19 esophageal adenocarcinoma cell line overexpressed HER2, and that both cell lines expressed PTEN (Fig. 3a). Flow cytometry also revealed that HER2 was strongly expressed on the cell surface in both the NCI-N87 cell line and the OE19 cell line (data not shown). The cell viability was examined with different concentrations of trastuzumab in 11 cell lines to determine trastuzumab sensitivity. Two HER2-overexpressing cell lines, NCI-N87 and OE19, were sensitive to trastuzumab at a concentration of $1 \mu \mathrm{g} / \mathrm{ml}$ (Fig. 3b).

PTEN knockdown was performed with nonsilencing control siRNA and two different sequences of siRNA targeting PTEN (referred to as "siPTEN \#1" and "siPTEN \#2") to investigate the influence of PTEN deficiency on trastuzumab sensitivity. PTEN expression was sufficiently knocked down in both the NCI-N87 cell line and the OE19 cell line at 72 and $96 \mathrm{~h}$ from transfection with siRNA (Fig. 3c, d). Further analyses were performed between 72 and $96 \mathrm{~h}$ after transfection.

The cell growth inhibition rate was calculated as the percentage reduction of cell viability when cells were treated with trastuzumab at $10 \mu \mathrm{g} / \mathrm{ml}$ for $72 \mathrm{~h}$ in comparison with untreated controls. The NCI-N87 cell line and the OE19 cell line both showed a significantly lower cell growth inhibition rate in PTEN-knockdown cells in comparison with cells with control siRNA $(19.3 \%$ and $19.5 \%$ vs $39.9 \%$ in the NCI$\mathrm{N} 87$ cell line, $p<0.01 ; 19.5 \%$ and $22.1 \%$ vs $34.1 \%$ in the OE19 cell line, $p<0.01$; Fig. 3e, f).

\section{PTEN knockdown reverses trastuzumab-induced Akt inactivation, inhibits apoptosis, and partially suppresses $\mathbf{G}_{1}$ cell cycle arrest}

The phosphorylation status of Akt and ERK, two key proteins in the PI3K and the MAPK downstream signaling pathways of HER2, was evaluated to investigate the mechanisms by which PTEN knockdown induces trastuzumab resistance. Phosphorylation of Akt and that of ERK were both considerably reduced in the nontransfected NCI-N87 and OE19 cells by treatment with trastuzumab at $10 \mu \mathrm{g} / \mathrm{ml}$ for $24 \mathrm{~h}$ (Fig. 4a). However, phosphorylation of Akt was mostly maintained in spite of trastuzumab treatment following PTEN knockdown with siPTEN \#1 and siPTEN \#2, whereas ERK was dephosphorylated to a similar extent as in cells transfected with nonsilencing control siRNA (Fig. 4a).

The relative caspase 3/7 activity, which represents apoptosis activity, following treatment with trastuzumab at $10 \mu \mathrm{g} /$ $\mathrm{ml}$ for $72 \mathrm{~h}$ was measured in comparison with that in the untreated control NCI-N87 and OE19 cells transfected with nonsilencing control or PTEN-targeting siRNAs. Although trastuzumab treatment increased the relative caspase 3/7 activity in both cell lines with nonsilencing control siRNA, the increases in the caspase activity with trastuzumab treatment were significantly lower in PTEN-knockdown cells (1.56 vs 1.13 and 1.14 in NCI-N87 cells, $p<0.01 ; 1.66$ vs 1.20 and 1.43 in OE19 cells, $p<0.01$; Fig. $4 b$ ).

The fraction of $G_{1}$ phase was significantly increased following treatment with trastuzumab at $10 \mu \mathrm{g} / \mathrm{ml}$ for $72 \mathrm{~h}$ in both NCI-N87 and OE19 cells with nonsilencing control siRNA $(69.8 \%$ vs $74.4 \%$ in NCI-N87 cells, $p<0.01$; $72.4 \%$ vs $75.3 \%$ in OE19 cells, $p<0.01$ ), indicating that trastuzumab induced $G_{1}$ cell cycle arrest. On the other hand, the proportion of $\mathrm{G}_{1}$ phase was not increased by trastuzumab treatment in the PTEN-knockdown NCI-N87 cells. Similar results were also obtained in OE19 cells, although a moderate increase of $\mathrm{G}_{1}$ phase was observed in cells with siPTEN \#1 (Fig. 4c).

\section{PTEN knockdown induces trastuzumab resistance in xenograft models}

We further explored the influence of PTEN knockdown on trastuzumab sensitivity using xenograft models. Stable PTEN-knockdown clones and scrambled control 


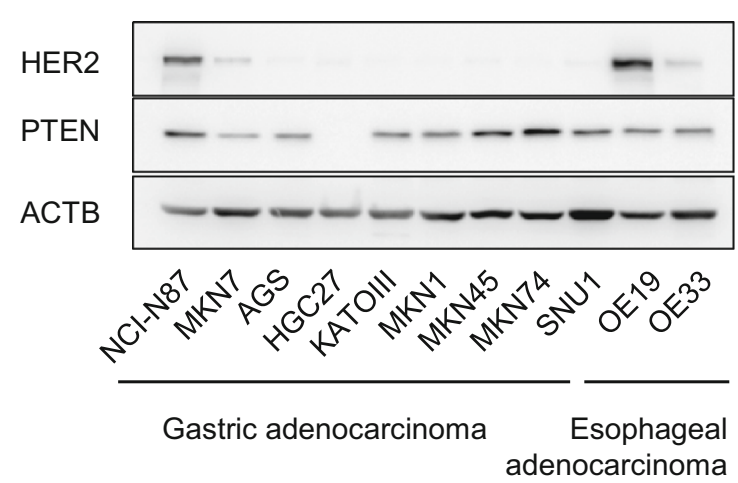

C

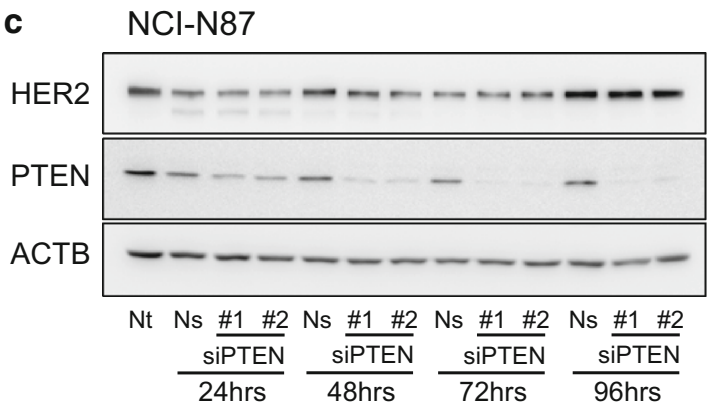

e

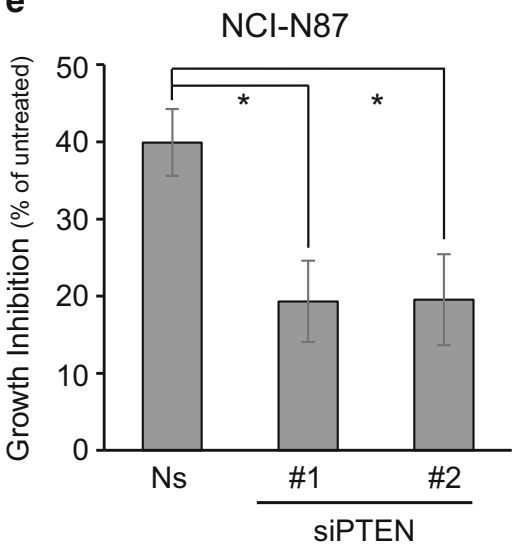

Fig. 3 Phosphatase and tensin homolog (PTEN) knockdown induces trastuzumab resistance in trastuzumab-sensitive cell lines. a Western blotting analyses of human epidermal growth factor receptor 2 (HER2) and PTEN in nine gastric adenocarcinoma cell lines and two esophageal adenocarcinoma cell lines. b Cell viability assays with increasing concentration of trastuzumab exposure for $72 \mathrm{~h}$ in 11 cell lines. PTEN knockdown was performed with two distinct small interfering RNA (siRNAs) targeting PTEN (siPTEN \#1 and siPTEN \#2), and the PTEN expression was analyzed by Western blotting at

clones were established by lentiviral infection of shRNAs and puromycin selection in NCI-N87 and OE19 cells. The PTEN expression was sufficiently knocked down in OE19 cells (Fig. 5a), whereas the PTEN knockdown was not sufficient in NCI-N87 cells (data not shown). The b
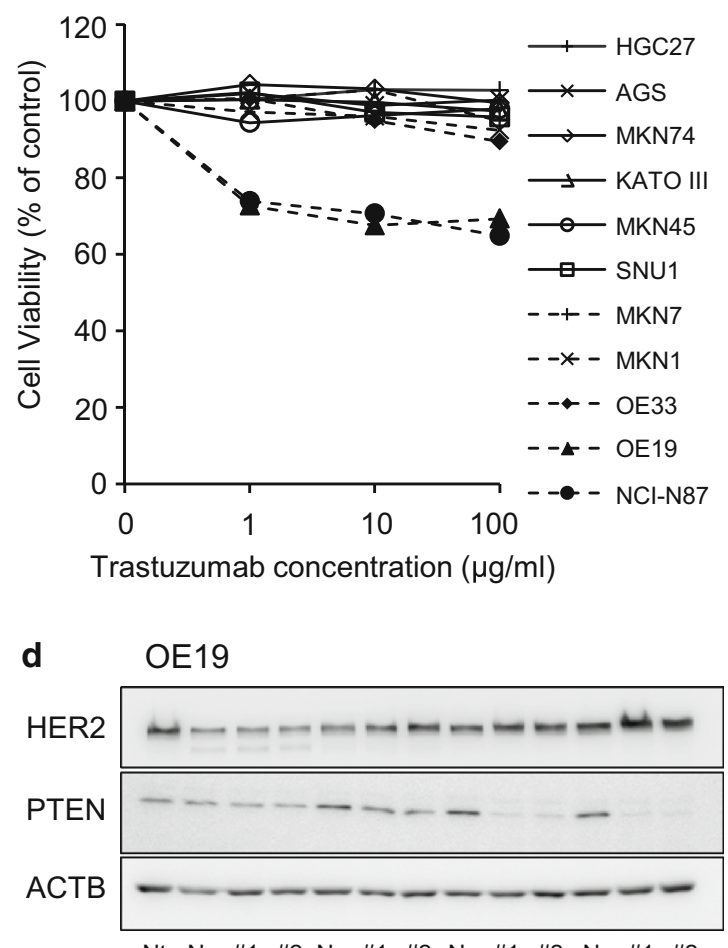

Nt Ns \#1 \#2 Ns \#1 \#2 Ns \#1 \#2 Ns \#1 \#2 $\frac{\text { siPTEN }}{24 \mathrm{hrs}} \frac{\overline{\text { siPTEN }}}{48 \mathrm{hrs}} \frac{\overline{\text { siPTEN }}}{72 \mathrm{hrs}} \frac{\text { siPTEN }}{96 \mathrm{hrs}}$

f

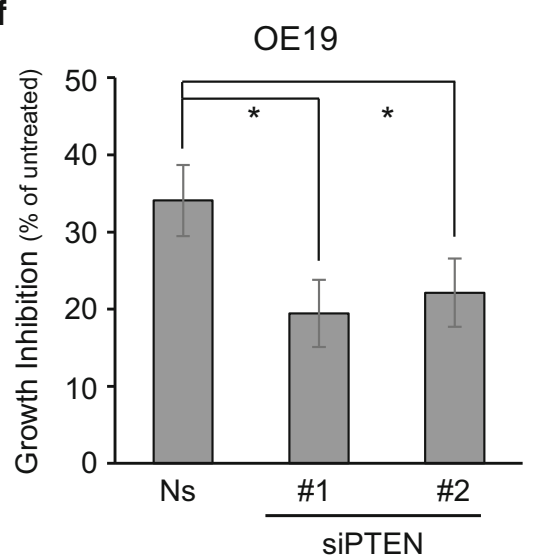

the indicated time points after transfection in the NCI-N87 cell line (c) and the OE19 cell line (d). Cells transfected with nonsilencing control siRNA $(N s)$, siPTEN \#1, or siPTEN \#2 were treated with trastuzumab $(10 \mu \mathrm{g} / \mathrm{ml})$ for $72 \mathrm{~h}$ in the NCI-N87 cell line (e) and the OE19 cell line (f). Growth inhibition rates were calculated as the percentage reduction of the cell viability in comparison with the untreated controls. Asterisk $p<0.01$, ACTB $\beta$-actin, Nt nontransfected control

scrambled control OE19 cells or stable PTEN-knockdown OE19 cells (referred to as shPTEN \#1 and shPTEN \#2) were inoculated subcutaneously, and treatment with intraperitoneal injection of normal saline or trastuzumab (30 mg/kg loading dose followed by $15 \mathrm{mg} / \mathrm{kg}$ weekly) 
Fig. 4 Phosphatase and tensin homolog (PTEN) knockdown reverses trastuzumab-induced Akt suppression, apoptosis, and $\mathrm{G}_{1}$ cell cycle arrest. a Western blotting analyses evaluating the influence of PTEN knockdown on phosphorylation of Akt and extracellular-signal-regulated kinase $(E R K)$. Nontransfected control cells and cells transfected with nonsilencing small interfering RNA (siRNA), siPTEN \#1, or siPTEN \#2 were not treated with trastuzumab or were treated with trastuzumab at $10 \mu \mathrm{g} / \mathrm{ml}$ for $24 \mathrm{~h}$. b Cells transfected with nonsilencing siRNA, siPTEN \#1, or siPTEN \#2 were not treated with trastuzumab or were treated with trastuzumab at $10 \mu \mathrm{g} / \mathrm{ml}$ for $72 \mathrm{~h}$. The caspase $3 / 7$ activity was measured with a Caspase-Glo 3/7 assay kit. The relative caspase $3 / 7$ activity of trastuzumab-treated cells in comparison with that of untreated cells was calculated in each cell line. c Flow cytometry analysis detecting the distribution of $G_{1}$ phase in the cell cycle. Cells transfected with nonsilencing siRNA, siPTEN \#1, or siPTEN \#2 were not treated with trastuzumab or were treated with trastuzumab at $10 \mu \mathrm{g} / \mathrm{ml}$ for $72 \mathrm{~h}$. Asterisk $p<0.01$, Ns nonsilencing siRNA, $N t$ nontransfected control
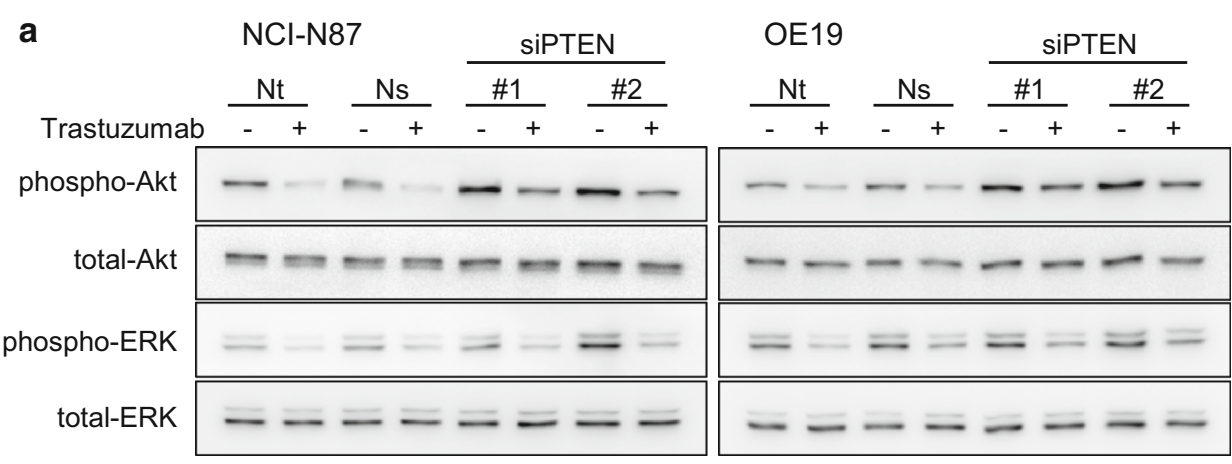

b
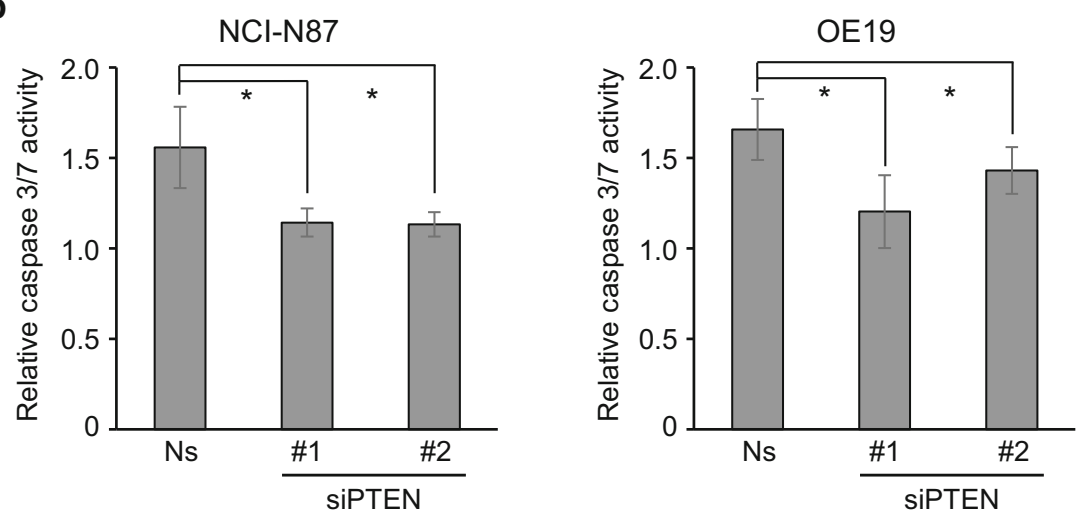

C

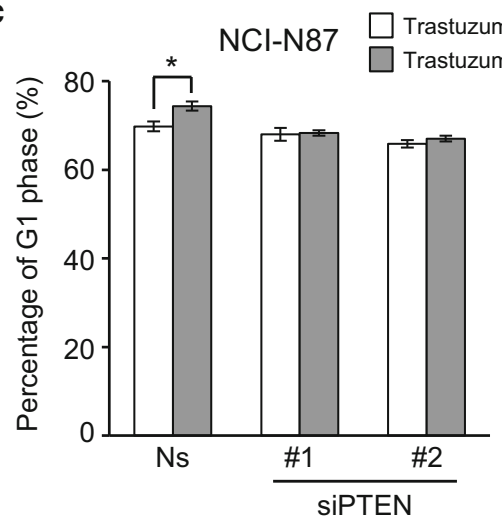

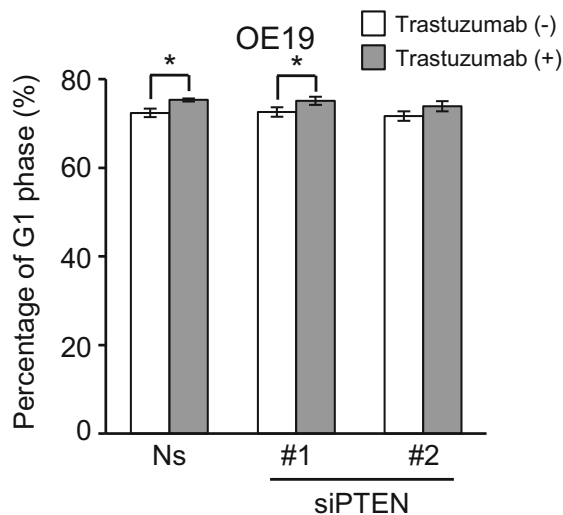

was started 2 weeks after inoculation, when tumors became palpable and measurable. Representative images of the xenograft tumors with normal saline treatment or trastuzumab treatment are shown in Fig. $5 \mathrm{~b}$ and c respectively. The tumor volume in the scrambled control tumors was significantly decreased in response to trastuzumab treatment in comparison with normal saline treatment (Fig. 5d; $p=0.035)$. In contrast, the proliferation of the tumors derived from PTEN-knockdown cells was not suppressed by trastuzumab treatment (Fig. 5e, f; $p=0.200$ and $p=0.886)$. HER2 expression and PTEN expression in xenograft tumor tissues after trastuzumab treatment were evaluated by IHC (Fig. 5g). Expression of both HER2 and PTEN was homogeneous in these tissues. HER2 expression was similar among scrambled control tumors and PTEN- knockdown tumors, whereas PTEN expression was strong in scrambled control tumors and negative in PTENknockdown tumors.

\section{Patients with PTEN loss show a poor response to trastuzumab-based therapy}

We further investigated the clinical significance of PTEN loss in trastuzumab therapy for GEA in the investigation cohort. Twenty-three patients who received trastuzumabbased therapy were examined. Nineteen of those patients were treated with capecitabine and cisplatin with trastuzumab, three patients were treated with S-1 and cisplatin with trastuzumab, and one patient was treated with capecitabine with trastuzumab. Table 2 shows that PTEN loss 


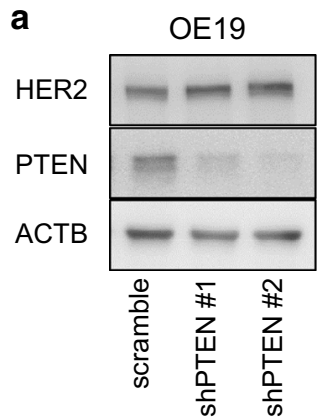

b

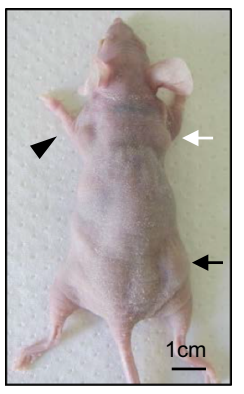

C

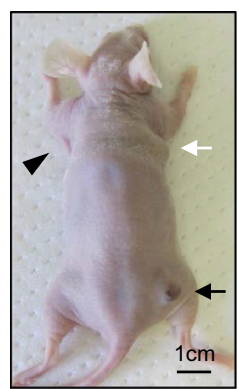

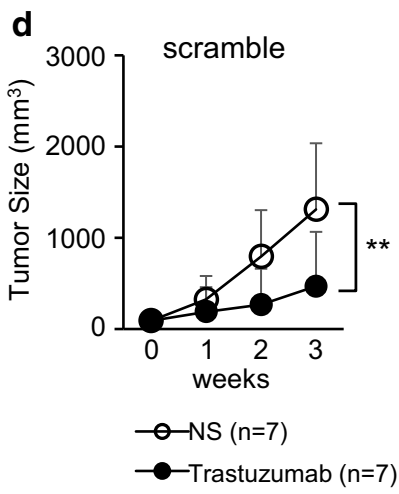
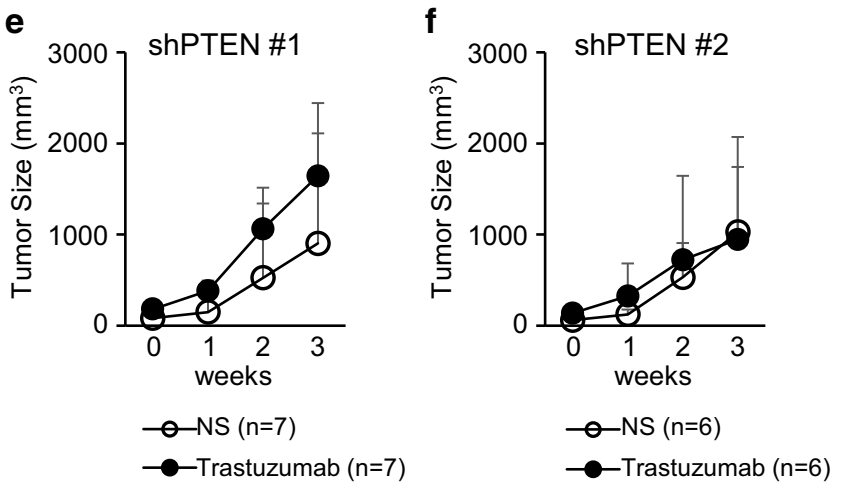

g

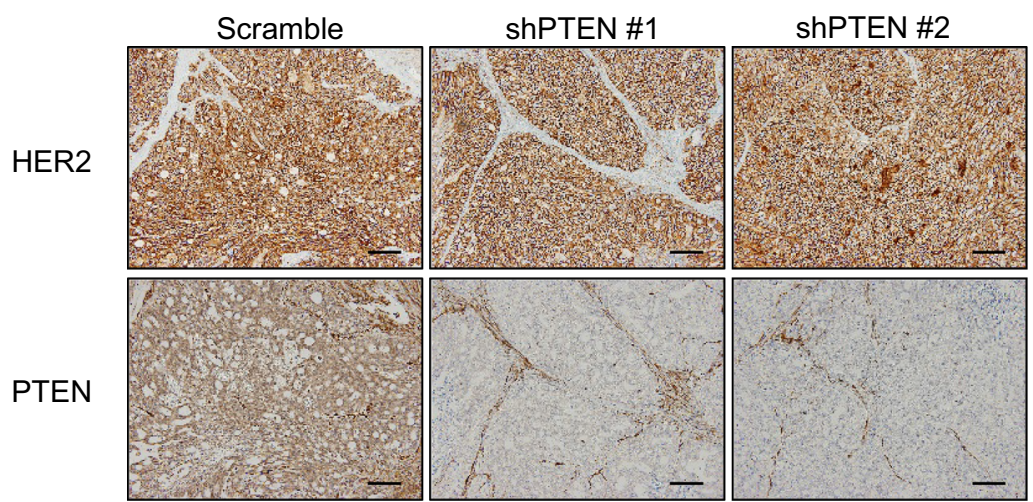

Fig. 5 Phosphatase and tensin homolog $(P T E N)$ knockdown induces trastuzumab resistance in xenograft models. a Stable PTEN-knockdown clones were selected and the PTEN expression was evaluated by Western blotting. Representative images of the xenograft model in intraperitoneal normal saline injection mice (b) and in intraperitoneal trastuzumab (30 mg/kg loading dose followed by $15 \mathrm{mg} / \mathrm{kg}$ weekly) injection mice (c). Arrowheads represent tumors with scrambled control, white arrows and black arrows represent tumors with shPTEN \#1 and shPTEN \#2 respectively. Scale bars represent $1 \mathrm{~cm}$. Tumor growth curves of xenografts derived from cells with scrambled

was found in five patients. None of these five patients with PTEN loss showed an objective clinical response to trastuzumab-based therapy. On the other hand, 9 of 18 PTENpositive patients $(50 \%)$ responded to the trastuzumabbased therapy, including one complete response and eight partial responses, although the difference in the response was not statistically significant between PTEN-loss patients and PTEN-positive patients (Table 2, $p=0.142$ ). control (d), shPTEN \#1 (e), and shPTEN \#2 (f). Mice were treated with weekly intraperitoneal injection of normal saline or trastuzumab. The data represent the mean tumor volume plus the standard deviation. $\mathbf{g}$ human epidermal growth factor receptor 2 (HER2) and PTEN immunohistochemistry images of scrambled control, shPTEN \#1, and shPTEN \#2 xenograft tumor specimens after trastuzumab treatment. In PTEN IHC, immunoreactivity of adjacent stromal cells was used as an internal positive control. Scale bars represent $100 \mu \mathrm{m}$. Two asterisks $p<0.05$, ACTB $\beta$-actin, NS normal saline

\section{Discussion}

Trastuzumab has significantly improved survival in patients with HER2-overexpressing GEA [5]. However, a limited response and resistance to trastuzumab-based therapy remain as obstacles. Only a few reports have examined the relationship between PTEN loss and trastuzumab resistance in gastric cancer [14-16], although PI3K 
Table 2 Phosphatase and tensin homolog (PTEN) expression and objective response to trastuzumab

\begin{tabular}{llll}
\hline Objective response & \multicolumn{2}{l}{ PTEN expression } & \multirow{2}{*}{$p^{\mathrm{a}}$} \\
\cline { 2 - 3 } & Positive $(n=18)$ & Loss $(n=5)$ & \\
\hline Complete response & 1 & 0 & 0.142 \\
Partial response & 8 & 0 & \\
Stable disease & 7 & 5 & \\
Progressive disease & 2 & 0 & \\
\hline
\end{tabular}

${ }^{a}$ Fisher's exact test

pathway alteration, including PTEN loss, is well known as an inducer of trastuzumab resistance in breast cancer [7-9]. The current study is the first to comprehensively investigate the impact of PTEN deficiency on trastuzumab response in HER2-overexpressing esophageal adenocarcinoma.

The current screening for PTEN loss and PIK3CA gene mutations, which involve two major aberrations of the PI3K pathway, revealed that $23.5 \%$ of patients showed PTEN loss and $9.6 \%$ showed PIK3CA mutations in a cohort of 264 curatively resected GEA patients. These findings are consistent with those of previous reports in gastric cancer (11-21.5\% with PTEN loss [17-19] and 2.5-15.9\% with PIK3CA mutations [18, 20-22]). More importantly, as many as $34.5 \%$ of patients with HER2 overexpression who are thought to be candidates for trastuzumab therapy showed PTEN loss, whereas PIK3CA mutations were found in only $5.6 \%$ of these patients. These data suggest the potential importance of PTEN loss in affecting the response to trastuzumab-based therapy in GEA.

Zaitsu et al. [19] showed that loss of heterozygosity of PTEN, which results in PTEN deficiency, was associated with HER2 overexpression. However, some reports have shown that loss of PTEN expression or deletion of the PTEN gene is rarely found (3.7-5.3 \%) in HER2-overexpressing gastric cancer $[23,24]$. The discrepancy in these results might be due to variations in the definition of PTEN loss. Thus, a validated standard definition of PTEN loss may be required to evaluate the influence of PTEN expression on the response to trastuzumab therapy.

HER2 expression in GEA is often heterogeneous, as we have previously reported [11]. Although HER2 expression was also highly heterogeneous in the current screening cohort, PTEN expression was relatively homogeneous (Fig. 2). A previous study also found that PTEN deletion was often homogeneous in gastric cancer [24]. Given the homogeneous distribution of PTEN-negative cells in GEA, evaluation of PTEN expression in a biopsy specimen will likely be a reliable method for evaluation of PTEN loss.
Trastuzumab suppresses the HER2 signaling of both the PI3K pathway and the MAPK pathway, and thus induces apoptosis and $G_{1}$ cell cycle arrest, which contribute to the antiproliferative effect [25]. The current study showed that the cell growth inhibition by trastuzumab was significantly suppressed in PTEN-knockdown cells, in which Akt remained phosphorylated despite trastuzumab treatment, and trastuzumab-induced apoptosis and $G_{1}$ cell cycle arrest were both prevented. These findings suggest that constitutively activated Akt by PTEN knockdown leads to cell survival and cell proliferation by inhibiting apoptosis and $\mathrm{G}_{1}$ cell cycle arrest, resulting in trastuzumab resistance. The importance of the inhibition of the PI3K pathway on the antitumor effect of trastuzumab should be different among different cell types, because trastuzumab can act via multiple mechanisms. These could explain why the degree of cell cycle inhibition by trastuzumab and its recovery by PTEN knockdown was slightly different between NCI-N87 and OE19 cells. Although $\mathrm{G}_{1}$ cell cycle arrest by trastuzumab was not completely restored by PTEN knockdown in OE19 cells in vitro, the antiproliferative effect of trastuzumab was completely abrogated by PTEN knockdown in xenograft models. These results imply that Akt activation by PTEN loss indeed plays a major role in the acquisition of resistance to trastuzumab in vivo.

The current clinical data indicate that patients with GEA with PTEN loss have a limited response to trastuzumabbased therapy. Zhang et al. [14] recently examined 48 gastric cancer patients with HER2 overexpression who clinically received anti-HER2 therapy (39 patients received trastuzumab therapy and 9 patients received lapatinib therapy), and showed that patients with PTEN loss had shorter progression-free survival than those with PTEN positivity in gastric cancer. These results and the current data support the finding that PTEN deficiency contributes to trastuzumab resistance.

The current study has several limitations. First, since the clinical data were retrospectively examined and the sample size was small, the results are only preliminary. However, of note, the findings from our functional experiments suggested that PTEN deficiency contributed to trastuzumab resistance through PI3K pathway activation in GEA cell lines. Second, we did not investigate the influence of PTEN knockdown on the combined effects of trastuzumab and other chemotherapeutic drugs, although trastuzumab is clinically approved for use in combination with other drugs for treatment of HER2-overexpressing GEA. However, several studies have shown that PTEN deficiency is also associated with increased resistance to multiple chemotherapeutic agents [16, 26]. Collectively, PTEN loss could be a clinically valuable biomarker of resistance to trastuzumab-based chemotherapy in GEA. It has been suggested that combination therapy with PI3K inhibitors 
might be helpful in overcoming PTEN-loss-mediated trastuzumab resistance in GEA [16] and in breast cancer $[7,27,28]$. These present and previous results suggest that patients with HER2-overexpressing GEA and PTEN loss might require alternative therapy to trastuzumab therapy, or combination therapy with PI3K inhibitors.

In conclusion, HER2-overexpressing GEA includes a considerable subset of patients with PTEN loss, and this is closely associated with a poor response to trastuzumabbased therapy. Further prospective studies are required to validate the predictive value of PTEN loss in trastuzumabbased therapy for patients with HER2-overexpressing GEA.

Acknowledgments We are grateful to Takahiro Horie for technical assistance in producing short hairpin RNA vectors, and Hiroyuki Shirahase for pathology technical support. We thank Chugai Pharmaceutical Co. Ltd for kindly providing trastuzumab. This work was supported by Japan Society for the Promotion of Science KAKENHI grant no. 24591940.

\section{Compliance with ethical standards}

Human rights statement and informed consent All procedures followed were in accordance with the ethical standards of the responsible committee on human experimentation (institutional and national) and with the Helsinki Declaration of 1964 and later versions. Informed consent was obtained from all patients for their being included in the study.

Animal studies All institutional and national guidelines for the care and use of laboratory animals were followed.

Conflict of interest The authors declare that they have no conflict of interest.

\section{References}

1. Ferlay J, Soerjomataram I, Dikshit R, Eser S, Mathers C, Rebelo $\mathrm{M}$, et al. Cancer incidence and mortality worldwide: sources, methods and major patterns in GLOBOCAN 2012. Int J Cancer. 2015;136(5):E359-86.

2. Yamada Y, Higuchi K, Nishikawa K, Gotoh M, Fuse N, Sugimoto N, et al. Phase III study comparing oxaliplatin plus S-1 with cisplatin plus S-1 in chemotherapy-naive patients with advanced gastric cancer. Ann Oncol. 2015;26(1):141-8.

3. Shah MA. Update on metastatic gastric and esophageal cancers. J Clin Oncol. 2015;33(16):1760-9.

4. Rubenstein JH, Shaheen NJ. Epidemiology, diagnosis, and management of esophageal adenocarcinoma. Gastroenterology. 2015;149(2):302-17.

5. Bang YJ, Van Cutsem E, Feyereislova A, Chung HC, Shen L, Sawaki A, et al. Trastuzumab in combination with chemotherapy versus chemotherapy alone for treatment of HER2-positive advanced gastric or gastro-oesophageal junction cancer (ToGA): a phase 3, open-label, randomised controlled trial. Lancet. 2010;376(9742):687-97.

6. Ades F, Zardavas D, Pinto AC, Criscitiello C, Aftimos P, de Azambuja E. Cardiotoxicity of systemic agents used in breast cancer. Breast. 2014;23(4):317-28.
7. Nagata Y, Lan KH, Zhou X, Tan M, Esteva FJ, Sahin AA, et al. PTEN activation contributes to tumor inhibition by trastuzumab, and loss of PTEN predicts trastuzumab resistance in patients. Cancer Cell. 2004;6(2):117-27.

8. Berns K, Horlings HM, Hennessy BT, Madiredjo M, Hijmans EM, Beelen K, et al. A functional genetic approach identifies the $\mathrm{PI} 3 \mathrm{~K}$ pathway as a major determinant of trastuzumab resistance in breast cancer. Cancer Cell. 2007;12(4):395-402.

9. Nahta R, Yu D, Hung MC, Hortobagyi GN, Esteva FJ. Mechanisms of disease: understanding resistance to HER2-targeted therapy in human breast cancer. Nat Clin Pract Oncol. 2006;3(5):269-80.

10. Eisenhauer EA, Therasse P, Bogaerts J, Schwartz LH, Sargent D, Ford R, et al. New response evaluation criteria in solid tumours: revised RECIST guideline (version 1.1). Eur J Cancer. 2009;45(2):228-47.

11. Kataoka Y, Okabe H, Yoshizawa A, Minamiguchi S, Yoshimura $\mathrm{K}$, Haga $\mathrm{H}$, et al. HER2 expression and its clinicopathological features in resectable gastric cancer. Gastric Cancer. 2013;16(1):84-93.

12. Bettstetter M, Berezowska S, Keller G, Walch A, Feuchtinger A, Slotta-Huspenina $\mathrm{J}$, et al. Epidermal growth factor receptor, phosphatidylinositol-3-kinase catalytic subunit/PTEN, and KRAS/NRAS/BRAF in primary resected esophageal adenocarcinomas: loss of PTEN is associated with worse clinical outcome. Hum Pathol. 2013;44(5):829-36.

13. Hartojo W, Silvers AL, Thomas DG, Seder CW, Lin L, Rao H, et al. Curcumin promotes apoptosis, increases chemosensitivity, and inhibits nuclear factor $\kappa \mathrm{B}$ in esophageal adenocarcinoma. Transl Oncol. 2010;3(2):99-108.

14. Zhang X, Park JS, Park KH, Kim KH, Jung M, Chung HC, et al. PTEN deficiency as a predictive biomarker of resistance to HER2-targeted therapy in advanced gastric cancer. Oncology. 2015;88(2):76-85.

15. Eto K, Iwatsuki M, Watanabe M, Ida S, Ishimoto T, Iwagami S, et al. The microRNA-21/PTEN pathway regulates the sensitivity of HER2-positive gastric cancer cells to trastuzumab. Ann Surg Oncol. 2014;21(1):343-50.

16. Zuo Q, Liu J, Zhang J, Wu M, Guo L, Liao W. Development of trastuzumab-resistant human gastric carcinoma cell lines and mechanisms of drug resistance. Sci Rep. 2015;5:11634.

17. Cho YJ, Kim JH, Yoon J, Cho SJ, Ko YS, Park JW, et al. Constitutive activation of glycogen synthase kinase- $3 \beta$ correlates with better prognosis and cyclin-dependent kinase inhibitors in human gastric cancer. BMC Gastroenterol. 2010;10:91.

18. Okines AF, Gonzalez de Castro D, Cunningham D, Chau I, Langley RE, Thompson LC, et al. Biomarker analysis in oesophagogastric cancer: results from the REAL3 and TransMAGIC trials. Eur J Cancer. 2013;49(9):2116-25.

19. Zaitsu Y, Oki E, Ando K, Ida S, Kimura Y, Saeki H, et al. Loss of heterozygosity of PTEN (encoding phosphate and tensin homo$\log$ ) associated with elevated HER2 expression is an adverse prognostic indicator in gastric cancer. Oncology. 2015;88(3):189-94.

20. Barbi S, Cataldo I, De Manzoni G, Bersani S, Lamba S, Mattuzzi $\mathrm{S}$, et al. The analysis of PIK3CA mutations in gastric carcinoma and metanalysis of literature suggest that exon-selectivity is a signature of cancer type. J Exp Clin Cancer Res. 2010;29:32.

21. Li VS, Wong CW, Chan TL, Chan AS, Zhao W, Chu KM, et al. Mutations of PIK3CA in gastric adenocarcinoma. BMC Cancer. 2005;5:29.

22. Moehler M, Mueller A, Trarbach T, Lordick F, Seufferlein T, Kubicka $\mathrm{S}$, et al. Cetuximab with irinotecan, folinic acid and 5-fluorouracil as first-line treatment in advanced gastroesophageal cancer: a prospective multi-center biomarker-oriented phase II study. Ann Oncol. 2011;22(6):1358-66. 
23. Zhang Z, Wang J, Ji D, Wang C, Liu R, Wu Z, et al. Functional genetic approach identifies MET, HER3, IGF1R, INSR pathways as determinants of lapatinib unresponsiveness in HER2-positive gastric cancer. Clin Cancer Res. 2014;20(17):4559-73.

24. Mina S, Bohn BA, Simon R, Krohn A, Reeh M, Arnold D, et al. PTEN deletion is rare but often homogeneous in gastric cancer. J Clin Pathol. 2012;65(8):693-8.

25. Nahta R, Esteva FJ. Herceptin: mechanisms of action and resistance. Cancer Lett. 2006;232(2):123-38.

26. Oki E, Baba H, Tokunaga E, Nakamura T, Ueda N, Futatsugi M, et al. Akt phosphorylation associates with LOH of PTEN and leads to chemoresistance for gastric cancer. Int $\mathrm{J}$ Cancer. 2005;117(3):376-80.

27. O'Brien NA, McDonald K, Tong L, von Euw E, Kalous O, Conklin D, et al. Targeting PI3K/mTOR overcomes resistance to HER2-targeted therapy independent of feedback activation of AKT. Clin Cancer Res. 2014;20(13):3507-20.

28. Lu CH, Wyszomierski SL, Tseng LM, Sun MH, Lan KH, Neal $\mathrm{CL}$, et al. Preclinical testing of clinically applicable strategies for overcoming trastuzumab resistance caused by PTEN deficiency. Clin Cancer Res. 2007;13(19):5883-8. 